\title{
Lead shock oscillation and decoupling in propagating detonations
}

\author{
J. M. Austin* \\ Aerospace Engineering, University of Illinois, Urbana-Champaign, IL 61801 USA \\ F.Pintgen and J.E. Shepherd \\ Graduate Aeronautical Laboratories, California Institute of Technology Pasadena, CA 91125 USA
}

\begin{abstract}
Experimental images of propagating detonation waves provide lead shock velocity measurements through the cell cycle. We examine the issue of local decoupling of the shock and reaction front using these data. In highly unstable mixtures with high reduced activation energy, experimental images and analysis suggest that local decoupling occurs at the end of the cell cycle. Regions of high fluorescence intensity are observed in shear layers in apparently decoupled portions of the detonation front.
\end{abstract}

\section{Introduction}

The coupling of the lead shock and the reaction front is critical to the propagation of near-CJ detonation waves. The reaction rate behind the shock is extremely sensitive to temperature perturbations and, as a result, gaseous detonation waves are always unstable. Past work into this instability has shown that the front is unsteady and the strength of the lead shock oscillates periodically.

The velocity of the lead shock through a cell cycle has been previously measured both experimentally $^{4,7,10,14,16}$ and numerically. ${ }^{6,8,15}$ Numerical simulations have directly shown that the excursion in the lead shock strength increases with increasing reduced activation energy. ${ }^{8}$

The present study contributes another set of experimental velocity measurements to the previous work, including a higher reduced activation energy mixture. In addition, we examine the possibility of local decoupling of the shock and reaction front based on analysis of the experimental data. We show how local decoupling may result in shear layers separating gas with different degree of reaction over a significant length over which the shear layer may become unstable. Experiments combining schlieren and planar laser induced fluorescence ${ }^{12}$ imaging of the shock and reaction front are reported. Images show regions of high fluorescence intensity in the shear layers near the end of the cell cycle for high reduced activation energy mixtures.

*Assistant Professor, Member AIAA

\section{Experimental measurements of lead shock propagation}
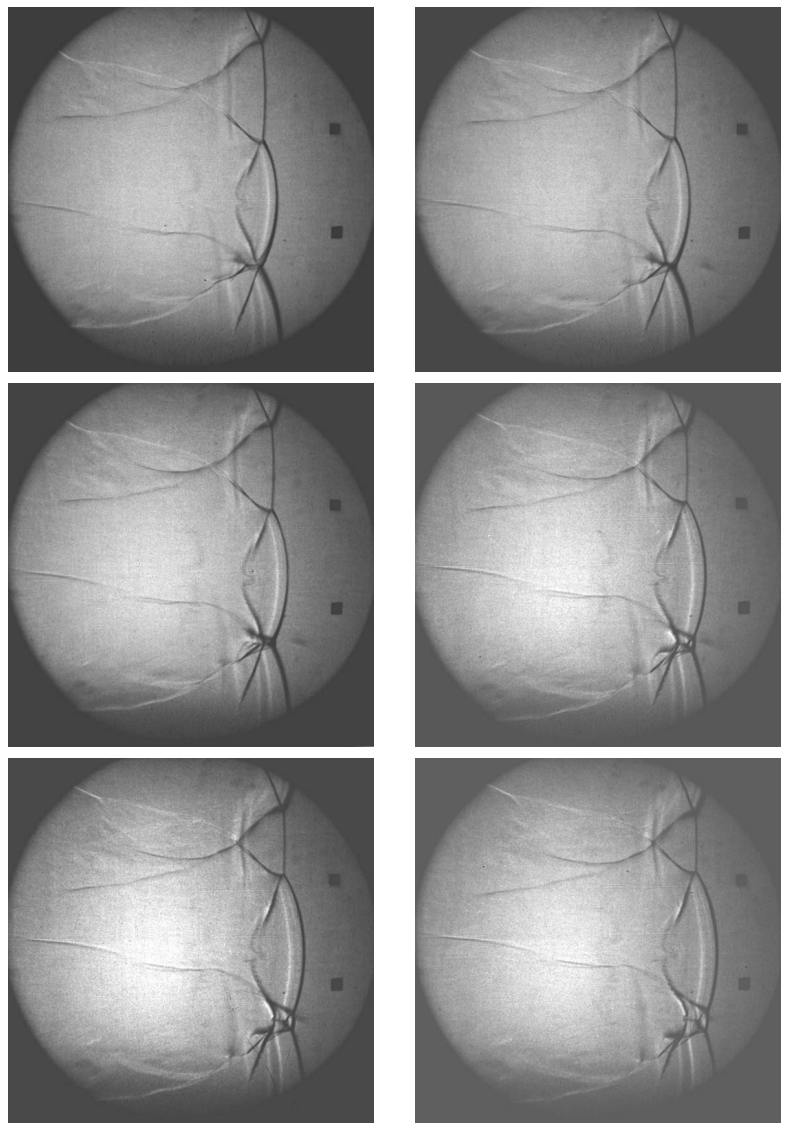

Fig. 1 Sample time-resolved shadowgraph images of a detonation propagating in $2 \mathrm{H}_{2}-\mathrm{O}_{2}-17 \mathrm{Ar}$, $P_{1}=20 \mathrm{kPa}$, in the narrow channel. Every second image acquired is shown, so the time between images is $1.7 \mu \mathrm{s}$. The field of view is $138 \mathrm{~mm}$.

High-speed shadowgraph movies were obtained of propagating detonation waves in a variety of fueloxidizer mixtures. Detonations were initiated directly $^{3,9}$ and propagated through a $4.2 \mathrm{~m}$ long high aspect ratio $(18 \times 152 \mathrm{~mm})$ rectangular channel. ${ }^{3}$ This design has the advantage of simplifying the flow field by reducing or entirely eliminating the transverse waves propagating in the direction of the smaller di- 
mension. Previous work by other researchers in similar channels has shown that there are some differences in the detonation structure from that observed in a rectangular channel. ${ }^{13,16}$ Pressure transducers were used to monitor the detonation wave speed along the channel. In all mixtures, detonations propagated at a constant velocity that was less than the CJ velocity. In Ar-diluted $\mathrm{H}_{2}-\mathrm{O}_{2}$ mixtures, the measured velocity was up to $8 \%$ less than the CJ velocity. In higher activation energy mixtures such as $\mathrm{C}_{3} \mathrm{H}_{8}-5 \mathrm{O}_{2}-9 \mathrm{~N}_{2}$, velocity deficits were less than $3 \%$. Please see Austin ${ }^{3}$ for more experimental details.

Multiple shadowgraph images per experiment were acquired using a Beckman and Whitley model 189 rotating mirror framing camera. Windows were located approximately $3.5 \mathrm{~m}$ downstream of the initiator. 25 images were acquired on Kodak TMAX 400 black and white $35 \mathrm{~mm}$ film. The time between frames is $832 \mathrm{~ns}$ with a 152 ns exposure time. A sample set of shadowgraph images of a weakly unstable detonation in $2 \mathrm{H}_{2}-\mathrm{O}_{2}-17 \mathrm{Ar}$ is shown in Fig. 1. The collision and refraction of two transverse waves occurs in the bottom right hand corner.

\section{Velocity measurements along the cell centerline}

Data from shadowgraph movies was compiled to create the $x$ - $t$ diagrams shown in Fig. 2 . The location of the lead shock is tracked along the cell centerline as a function of time. Error bars in position and time due to frame superposition and camera speed are $\pm 1 \mathrm{~mm}$ and $\pm 0.02 \mu s$ respectively. Results from three mixtures are shown: two marginally unstable cases with low reduced activation energy $\left(2 \mathrm{H}_{2}-\mathrm{O}_{2}-17 \mathrm{Ar}, 2 \mathrm{H}_{2}-\mathrm{O}_{2}-12 \mathrm{Ar}\right)$, and a highly unstable case with higher reduced activation energy $\left(\mathrm{C}_{3} \mathrm{H}_{8}-5 \mathrm{O}_{2}-9 \mathrm{~N}_{2}\right)$.

Power law curve fits, $\mathrm{t}=\mathrm{ax}^{b}$, were calculated for the experimental data using the least squares technique. Curves are shown in Fig. 2 and the resulting fit parameters, together with goodness of fit data are given in Table 1. The Taylor-Sedov (strong shock )similarity solution predicts $\mathrm{t}=\mathrm{Ar}^{5 / 2}$ for a spherical geometry and $\mathrm{t}=\mathrm{Ar}^{2}$ for a cylindrical geometry . In the present data, the shock decays faster than the Taylor-Sedov model.

The curve fits to the experimental $x$ - $t$ data were differentiated to obtain the lead shock velocity along the cell centerline, Fig. 3. Since the results of the power law fit are sensitive to the averaging process used, several piecewise fits were also made. The length of the piecewise region was varied and the resulting envelope of reasonable values is shown as a grey region in the figure. Data from the numerical study of Gamezo et al. ${ }^{8}$ for low and high reduced activation energy mixtures is also shown.

Based on the global curve fit, the lead shock velocity decays from about $1.5 U_{C J}$ to $0.8 U_{C J}$ through the cell cycle in $2 \mathrm{H}_{2}-\mathrm{O}_{2}-12 \mathrm{Ar}$. However, the local (piece-

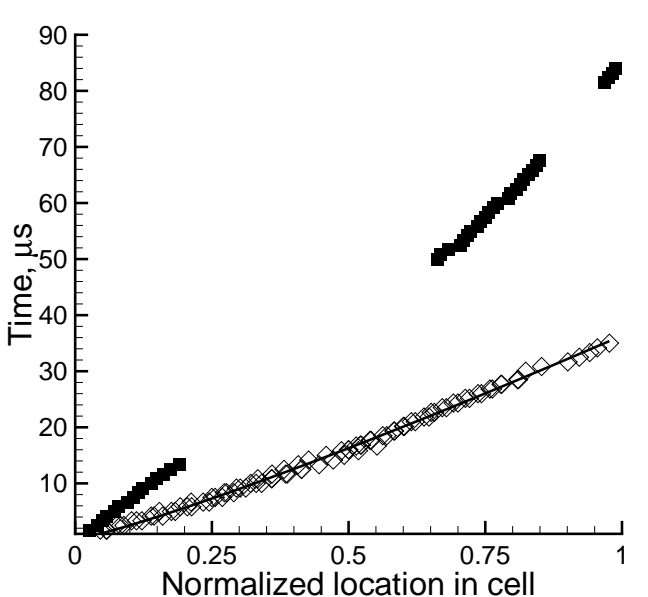

(a)

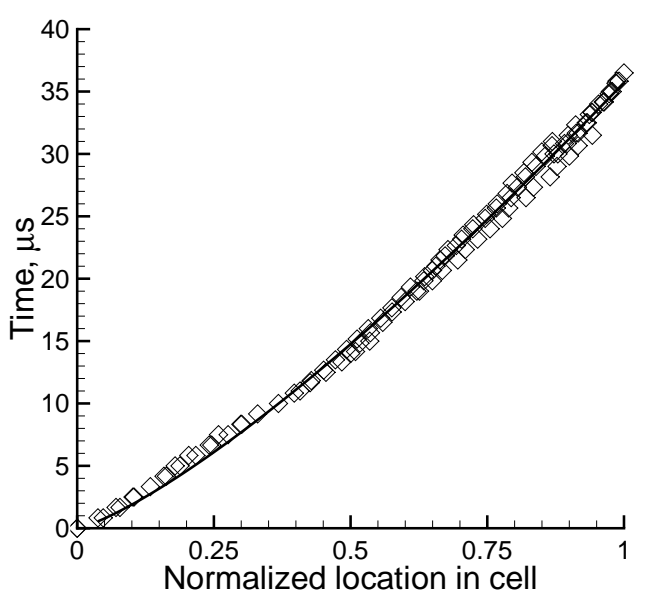

(b)

Fig. $2 x$ - $t$ diagrams of lead shock location through a cell cycle. (a) open symbols: $2 \mathrm{H}_{2}-\mathrm{O}_{2}-12 \mathrm{Ar}$, filled symbols: $2 \mathrm{H}_{2}-\mathrm{O}_{2}-17 \mathrm{Ar}$ and (b) $\mathrm{C}_{3} \mathrm{H}_{8}-5 \mathrm{O}_{2}$ $9 \mathrm{~N}_{2}$. Data from several experiments is presented. Note the time origin is arbitrary for each data set.

wise) curve fit over the interval $0<\left(x-x_{o}\right) / L<0.2$ results in a velocity near the cell apex of $1.2 \mathrm{U}_{C J}$. The magnitude of the fluctuation is then in good agreement with previous data. Lundstrom and Oppenheim measured 1.7-1.3<U/U $U_{C J}<0.8-0.6$. Dormal et al. ${ }^{4}$ and Strehlow and Crooker ${ }^{13}$ both report $1.2<U / U_{C J}<0.8$. In $\mathrm{C}_{3} \mathrm{H}_{8}-5 \mathrm{O}_{2}-9 \mathrm{~N}_{2}$, the lead shock velocity decays from about $1.9 U_{C J}$ to $0.8 U_{C J}$ based on the global curve fit. The local (piecewise) curve fit over the interval $0<\left(x-x_{o}\right) / L<0.2$, results in a velocity near the cell apex of $1.5 \mathrm{U}_{C J}$. The local fit values are probably more reasonable. Overall, the oscillation amplitude in the centerline velocity for the highly unstable mixture is is greater than for weakly unstable detonation. 


\begin{tabular}{|ccc|}
\hline Parameter & $2 \mathrm{H}_{2}-\mathrm{O}_{2}-12 \mathrm{Ar}$ & $\mathrm{C}_{3} \mathrm{H}_{8}-5 \mathrm{O}_{2}-9 \mathrm{~N}_{2}$ \\
\hline $\mathrm{a}$ & 35.71 & 36.34 \\
$\mathrm{a}_{L}$ & 35.45 & 36.02 \\
$\mathrm{a}_{U}$ & 35.98 & 36.67 \\
$\mathrm{~b}$ & 1.275 & 1.158 \\
$\mathrm{~b}_{L}$ & 1.251 & 1.141 \\
$\mathrm{~b}_{U}$ & 1.300 & 1.175 \\
$\mathrm{SSE}$ & 47.05 & 20.86 \\
$\mathrm{R}^{2}$ & 0.9959 & 0.9974 \\
$\mathrm{RMSE}$ & 0.6454 & 0.4762 \\
\hline
\end{tabular}

Table 1 Power law curve fits, $\mathbf{t}=a \mathbf{x}^{b}$, were calculated for a low and high reduced activation energy mixture using the least squares technique. Subscript $L$ and $U$ refer to the lower and upper limits of the $95 \%$ confidence level in calculating the parameter.SSE is the sum of the squares due to error (residuals). $\mathbf{R}^{2}$ is the square of the multiple correlation coefficient and indicates the total variation of the fit about the average. RMSE is the root mean square error.

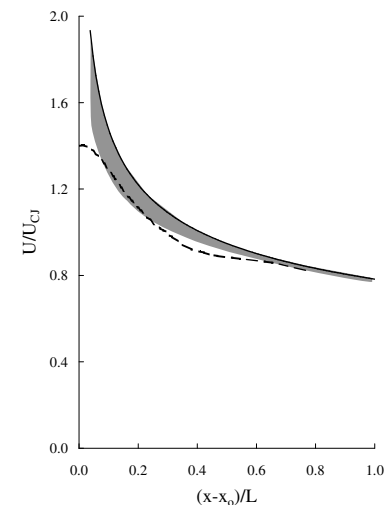

(a)

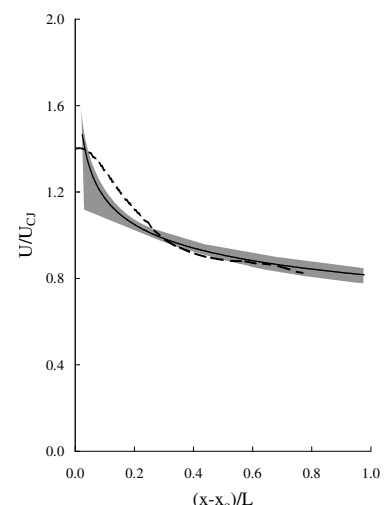

(b)
Fig. 3 Velocity of the lead shock front along the centerline of a cell. The solid line is obtained by differentiating the curve fit to the $x-t$ data over the entire length of the cell. Piecewise curve fits over different intervals were also made. These curves result in an envelope of reasonable values that is represented by the grey region. Dashed lines are data from the numerical study of Gamezo et al. ${ }^{8}$ for a reduced activation energies of a) 4.9 and b) 7.4 .

Local decoupling of the lead shock and reaction front

The velocity profile of the lead shock along the cell centerline through one cell cycle may be compared to that of a decaying blast wave due to a release of energy at the triple point collision at the beginning of a cell. ${ }^{10,16}$ An analogy may be made with detonation initiation by a strong, spherical blast wave. In that situation, two outcomes are possible. Successful initiation occurs when the strong blast wave decays approximately to the CJ velocity and becomes a self- sustained spherical detonation wave. Failed initiation occurs when the shock and reaction decouple and the blast wave continues to decay to an acoustic wave.

We investigate the possibility of decoupling of the decaying lead shock and the reaction along the centerline of one cell cycle using the the critical decay rate (CDR) model. The CDR model was developed by Eckett, Quirk and Shepherd ${ }^{5}$ and includes the effect of unsteadiness on the induction time. Their results were extended to more general wave geometries by Arienti. ${ }^{1}$ The key result of Eckett ${ }^{6}$ is that the curvature term was found to be negligible and a local criterion for detonation decoupling was found as a balance between the chemical energy release term and the unsteadiness terms. Failure occurs if the shock decay time $t_{d}$ is less than a critical value $t_{d, c}$ where

$$
\begin{aligned}
\frac{1}{t_{d}} & =-\frac{1}{U} \frac{d U}{d t}, \\
t_{d, c} & =6 \frac{\gamma-1}{\gamma+1} \theta \tau .
\end{aligned}
$$

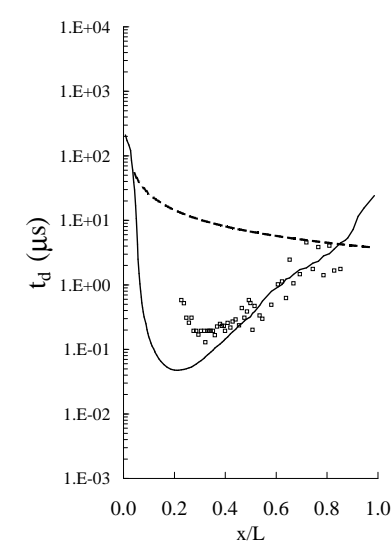

(a)

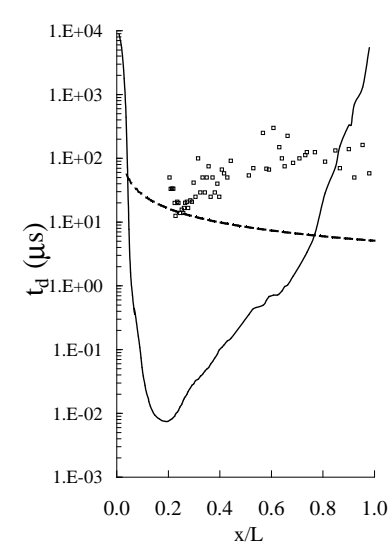

(b)
Fig. 4 Shock decay time vs. critical decay time for (a) low reduced activation energy mixtures: $2 \mathrm{H}_{2}-\mathrm{O}_{2}-12 \mathrm{Ar}$ with $\theta=5.2$ (dashed line) and numerical data $^{8}$ for $\theta=4.9$ (symbols) and (b) high reduced activation energy mixtures, $\mathrm{C}_{3} \mathrm{H}_{8}-5 \mathrm{O}_{2}-9 \mathrm{~N}_{2}$ with $\theta=12.7$ (dashed line) and numerical data ${ }^{8}$ for $\theta=7.9$ (symbols). The critical decay time from the CDR model ${ }^{5}$ is shown as the solid line.

This model has been applied by Pintgen et al. ${ }^{11}$ to weakly unstable detonation fronts using data from numerical simulations. ${ }^{6}$ It was found that decoupling of the detonation does not occur. In the present study, we use lead shock velocities along the cell centerline from current experiments and from the numerical simulations of Gamezo et al. ${ }^{8}$ which considered the effect of activation energy. Shock decay times are calculated and compared to the critical value from the CDR model. Results are shown in Fig. 4 for modest and high reduced activation energy mixtures. In the case of modest reduced activation energies $(\theta \sim 5)$, 
Fig. 4(a), the curve fit to the experimental data shows a larger decay time than the numerical study. In both cases, the decay time becomes comparable to the critical value at the end of the cell. The curve fit to the experimental data becomes slightly less than the critical value for $x / L>0.85$. For higher reduced activation energy cases, Fig. 4(b), the decay time is substantially less than the critical value at about $x / L>0.75$ and the model predicts local decoupling, or quenching, of the detonation wave.

\section{Mixing of gas with different degrees of reaction}

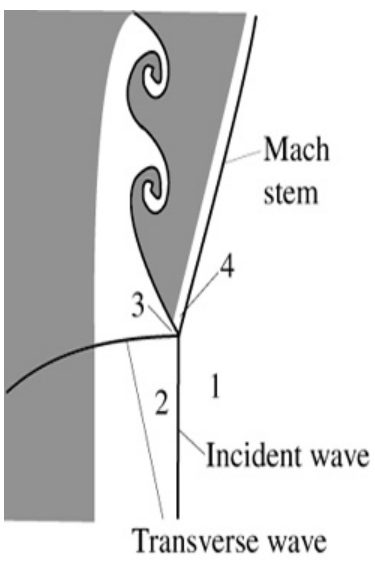

(a)

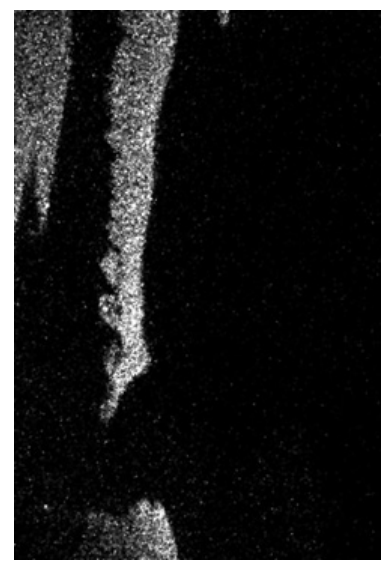

(b)
Fig. 5 a) Cartoon showing states at triple point configuration. b)Image of unstable shear layer separating gas of different degree of reaction $2 \mathrm{H}_{2}-\mathrm{O}_{2}$ $7.7 \mathrm{~N}_{2}, \mathrm{P}_{1}=20 \mathrm{kPa}$. Image ${ }^{3}$ was obtained using $\mathrm{OH}$ PLIF $^{12}$ in a $280 \mathrm{~mm}$ diameter detonation tube.

As reported in Pintgen et al., ${ }^{11}$ "keystones" are formed in weakly unstable fronts due to spatial oscillations in the lead shock strength. Triple point analysis showed that the shear layers form the boundary between fluid particles that have passed through the strong portion of the lead shock front (Mach stem) and react relatively quickly, and particles that have passed through the weak portion of the front (incident wave) and the transverse wave and react more slowly. In some portions of the front, the leading (incident) shock will be near the end of the cell cycle and there will be a large difference in the induction time of the gas on either side of the shear layer. An estimate of the difference in induction times across the shear layer based on a triple point analysis is shown in Table 2. Local decoupling of the shock and reaction zone would substantially increase the induction time disparity above these calculated values.

Shear layer instabilities are observed in schlieren images of weakly unstable fronts. In Fig. 6 (a), the shear layer attached to the leading shock (current cycle) appears unstable. In Fig. 6 (b), shear layer (previous cycle) appears unstable while in Fig. 6 (a), the

\begin{tabular}{|cccccc|}
\hline Mixture & $\Delta P / P_{2}$ & $\mathrm{u}_{3}$ & $\mathrm{u}_{4}$ & $\tau_{3}$ & $\tau_{4}$ \\
\hline $2 \mathrm{H}_{2}-\mathrm{O}_{2}-17 \mathrm{Ar}$ & 0.32 & 623 & 390 & 5.8 & 2.7 \\
$2 \mathrm{H}_{2}-\mathrm{O}_{2}-5.6 \mathrm{~N}_{2}$ & 0.37 & 716 & 352 & 7.9 & 2.5 \\
$\mathrm{C}_{3} \mathrm{H}_{8}-5 \mathrm{O}_{2}-9 \mathrm{~N}_{2}$ & 0.39 & 554 & 269 & 5.8 & 1.6 \\
\hline
\end{tabular}

Table 2 Calculated properties across a triple point contact surface for sample mixtures from this study. $\Delta P / P_{2}=\left(P_{3}-P_{2}\right) / P_{2}$ is the normalized pressure jump across the transverse wave. State 3 is behind the incident and transverse waves and state 4 is behind the Mach stem (Schematic is shown in Fig. 5). The triple point configuration is calculated using shock polars. The incident wave velocity wave is taken to be $0.9 U_{C J}$, representative of conditions near the end of the cell and the track angle is assumed to be $33^{\circ}$. Induction times $\tau$ are then calculated by taking particle paths through the three-shock configuration and using a constant volume explosion assumption and the detailed Konnov mechanism. ${ }^{3}$

shear layer from the previous cycle appears to become unstable after interaction with the transverse wave. The instability is not evident in the corresponding fluorescence image. In weakly unstable fronts, several hundred experiments have shown no shear layer instabilities in fluorescence images to the resolution of the study (at best $50 \mu \mathrm{m} /$ pixel).

In highly unstable fronts, regions of intense chemiluminescence have been observed (Fig. 7 a). From the associated schlieren images(Fig. 7 b), we can determine these bright regions correspond with the location

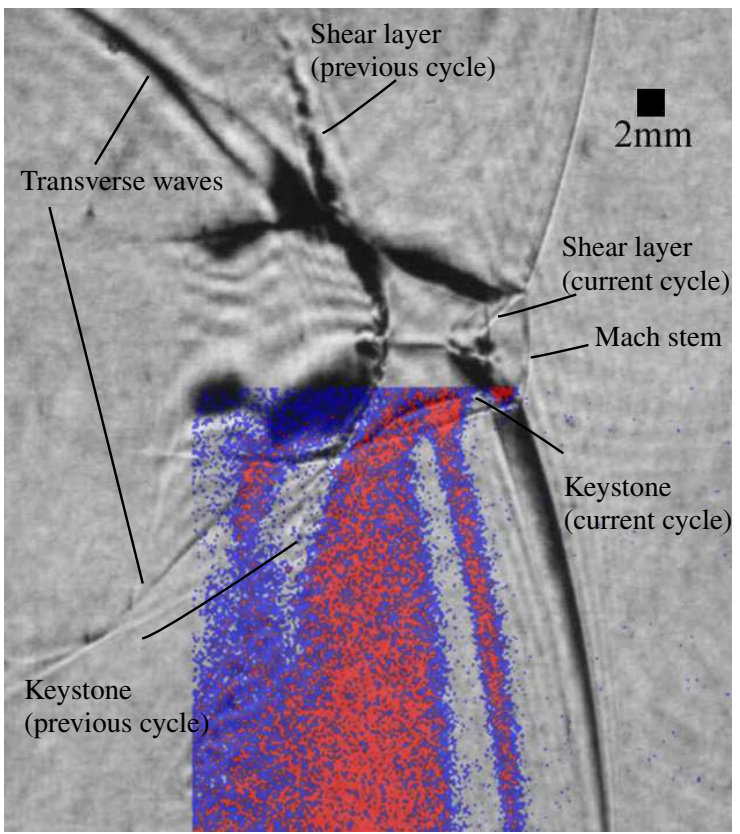

Fig. 6 Overlaid PLIF ${ }^{12}$ (false color) and schlieren images of portion of detonation front propagating left to right in $2 \mathrm{H}_{2}-\mathrm{O}_{2}-17 \mathrm{Ar}, P_{1}=20 \mathrm{kPa}^{3}$ 


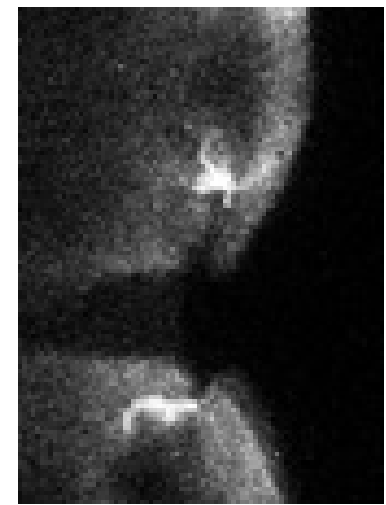

(a)

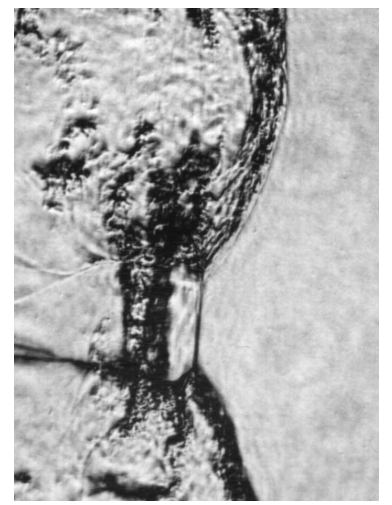

(b)
Fig. 7 a) Hot spots in chemiluminescence images in $\mathrm{C}_{3} \mathrm{H}_{8}-5 \mathrm{O}_{2}-9 \mathrm{~N}_{2}, P_{1}=20 \mathrm{kPa}$. Image height is $65 \mathrm{~mm}$. Regions of intense luminescence are observed in the shear layers near the end of the cell cycle. b) Schlieren image of the same portion of the front shown in a).

of shear layers. The detonation appears to have locally decoupled at the end of the cell cycle and the transverse wave collision has not yet occurred. If the shock and reaction front decouples at the end of the cell, the shear layer separates reacted and unreacted gas over a considerable length. With the increase in surface area in an unstable shear layer, we may speculate that local "hot spots" may be formed due to mixing of hot products and cold reactants, and possibly contribute to the re-ignition process at the end of the cell. This is a "turbulent" combustion mechanism that is not included in traditional detonation models based on shock-induced explosion.

\section{Conclusions}

High-speed shadowgraph data are used to create $x$ - $t$ diagrams of lead shock location through a cell cycle in propagating detonation waves. The velocity of the lead shock is calculated based on power law curve fits to this data. The magnitude of the lead shock oscillation is estimated to be about $1.2<U / U_{C J}<0.8$ in $2 \mathrm{H}_{2^{-}}$ $\mathrm{O}_{2}-12 \mathrm{Ar}$, which agrees with existing data for modest reduced activation energy mixtures. In a high reduced activation energy mixture, $\mathrm{C}_{3} \mathrm{H}_{8}-5 \mathrm{O}_{2}-9 \mathrm{~N}_{2}$, the magnitude of the lead shock oscillation increases to $1.5<U / U_{C J}<0.8$. This is consistent with the observations of Gamezo et al. ${ }^{8}$

The effect of the unsteadiness of the front on the coupling between the lead shock and the reaction is examined using the critical decay rate model. ${ }^{5}$ Based on the curve fit to the experimental data, the model predicts local decoupling, or quenching, of the portion of the detonation front near the end of the cell cycle $\left(\left(x-x_{o}\right) / L>0.75\right.$ for $\left.\mathrm{C}_{3} \mathrm{H}_{8}-5 \mathrm{O}_{2}-9 \mathrm{~N}_{2}\right)$.

Shear layers separate gas of different degree of reactivity at the triple points, forming keystone features in images of the reaction front. ${ }^{11}$ Increasing the magnitude of the lead shock oscillation through a cell cycle increases the magnitude of the disparity in induction time across the shear layer. Local decoupling of the front would increase this difference. In highly unstable mixtures, shear layer instability is observed in the region where the shear layer separates reacted and unreacted gas. We speculate that this creates the possibility for mixing of hot products and cold reactants and the formation of local "hot spots" that may play a role in the propagation of the detonation. Experimental images of regions of high fluorescence intensity and localized explosion ${ }^{2}$ in these portions of the front support this idea.

\section{References}

${ }^{1}$ Marco Arienti. A Numerical and Analytical Study of Detonation Diffraction. PhD thesis, California Institute of Technology, Pasadena, California, January 2003.

${ }^{2}$ J.M. Austin, F. Pintgen, and J.E. Shepherd. Reaction zones in highly unstable detonations. In Proceedings of the Combustion Institute, pages 1845-1853, Chicago, IL, 2004.

${ }^{3}$ Joanna M. Austin. The Role of Instability in Gaseous Detonation. PhD thesis, California Institute of Technology, Pasadena, California, May 2003.

${ }^{4}$ M. Dormal, J. C. Libouton, and P. Van Tiggelen. Evolution of induction time in detonation cells. Acta Astronautica, 6(1):875-88, 1979

${ }^{5}$ C. A. Eckett, J. J. Quirk, and J. E. Shepherd. The role of unsteadiness in direct initiation of gaseous detonation. J. Fluid Mechanics, 421:147-183, 2000.

${ }^{6}$ Christopher A. Eckett. Numerical and Analytical Studies of the Dynamics of Gaseous Detonations. PhD thesis, California Institute of Technology, Pasadena, California, September 2000.

${ }^{7}$ D. H. Edwards, G. Hooper, E. M. Job, and D. J. Parry. The behavior of the frontal and transverse shocks in gaseous detonation waves. Astronautica Acta, 15:323-333, 1970.

${ }^{8}$ V. N. Gamezo, D. Desbordes, and E. S. Oran. Twodimensional reactive flow dynamics in cellular detonation waves. Shock Waves, 9:11-17, 1999.

${ }^{9}$ S. I. Jackson and J. E. Shepherd. Initiation systems for pulse detonation engines. Technical Report AIAA 2002-3627, 38th AIAA/ASME/SAE/ASEE Joint Propulsion Conference, Indianapolis, IN, July 7-10 2002.

${ }^{10}$ E. A. Lundstrom and A. K. Oppenheim. On the influence of non-steadiness on the thickness of the detonation wave. Proc. Roy. Soc. A, 310:463-478, 1969.

${ }^{11}$ F. Pintgen, C. A. Eckett, J. M. Austin, and J. E. Shepherd. Direct observations of reaction zone structure in propagating detonations. Combust. Flame (in press), 2003.

${ }^{12}$ Florian Pintgen. Laser-Optical Visualization of Detonation Structures. Diplom Arbeit, Lehrstuhl für Thermodynamik: Technische Universität München / Graduate Aeronautical Laboratories: California Institute of Technology, December 2000.

${ }^{13}$ R.A. Strehlow and A.J. Crooker. The structure of marginal detonation waves. Acta Astronautica, 1:303-315, 1974.

${ }^{14}$ V.A. Subbotin. Two kinds of transverse wave structures in multi-front detonation. Fizika Goreniya $i$ Vzryva, 11(1):96-102, 1975 .

${ }^{15}$ R. Takai, K. Yoneda, and T. Hikita. Study of detonation wave structure. In 15th Symposium (International) on Combustion, pages 69-78, Pittsburgh, PA, 1974. The Combustion Institute.

${ }^{16}$ B. V. Voitsekhovskii, V. V. Mitrofanov, and M. E. Topchian. Struktura fronta detonastii i gaza. Akad. Nauk., SSSR, Novosibirsk, 1963. 